\title{
SOLAR AND INTERPLANETARY MAGNETIC FIELDS AND PLASMAS*
}

\author{
JoHN M. WILCOX \\ (Space Sciences Laboratory, University of California, \\ Berkeley, Calif., U.S.A.)
}

\begin{abstract}
ABST RACT
A few recent observations of interplanetary fields and plasmas are discussed, including the evolution over several years of the longitudinal sector pattern, the corotating filamentary structure that exists within the sectors, guiding of solar cosmic rays along such magnetic filaments, a field-aligned thermal anisotropy of the plasma, and a small component of corotating plasma velocity observed at $1 \mathrm{AU}$. The solar wind plasma streaming past spacecraft near $1 \mathrm{AU}$ contains a large amount of information about the detailed structure of the Sun.
\end{abstract}

\section{Introduction}

The use of satellites and space probes in the last few years to make in situ observations of interplanetary magnetic fields and plasmas has produced significant new information about the extended solar corona. Hydrodynamic expansion of the corona with a concomitant extension of the solar magnetic field was discussed theoretically by Parker (1963), and verified in 1962 by the Mariner-2 spacecraft on its flight to Venus. The velocity of the expanding solar plasma (solar wind) is approximately radial with a magnitude near $1 \mathrm{AU}$ of $350-400 \mathrm{~km} / \mathrm{sec}$ in quiet times, increasing to $600-700 \mathrm{~km} / \mathrm{sec}$ in disturbed intervals. The flux is about $10^{9} \mathrm{ions} / \mathrm{cm}^{2} / \mathrm{sec}$, the principal positive ionic component being protons with an energy of about $1 \mathrm{keV}$. A varying admixture of alpha particles is present with the average concentration about $5 \%$.

The combination of radial plasma velocity and solar rotation stretches the interplanetary magnetic field into an Archimedes spiral making an angle near the Earth of about $45^{\circ}$ with the Earth-Sun direction. The field near the Earth is approximately parallel to the ecliptic, and its average magnitude is about $6 y\left(6 \times 10^{-5}\right.$ gauss $)$, with a range from $2 \gamma$ to about $40 \gamma$, the larger values usually occurring during flare-associated disturbances.

\section{Evolution of Sector Structure}

This review of new observations will begin with a large-scale property of the interplanetary medium, and then proceed to smaller-scale structures. In accord with the

* This paper was prepared while the author was a guest at the Division of Plasma Physics, The Royal Institute of Technology, Stockholm, Sweden.

Perek (ed.), Highlights of Astronomy, 124-135. (C) I.A.U. 
purpose of this symposium only a few recent observations are discussed; a complete survey is not attempted (for a recent review see Ness, 1967). An unexpected property of the interplanetary magnetic field is shown in Figure 1, which gives the predominant polarity (toward or away from the Sun) of the field observed by IMP-1 during the winter of 1963-64. For several consecutive days the field polarity remains unidirectional. The sector pattern thus defined corotates with the Sun; i.e., the pattern tends to repeat itself every 27 days. Considerable evidence (Wilcox and Ness, 1965) suggests that each sector so defined was a coherent entity, with plasma velocity and field magnitude reaching maxima in the preceding portion and declining in the following portion. Observations from Mariner-2 had previously shown a pattern of recurring maxima in

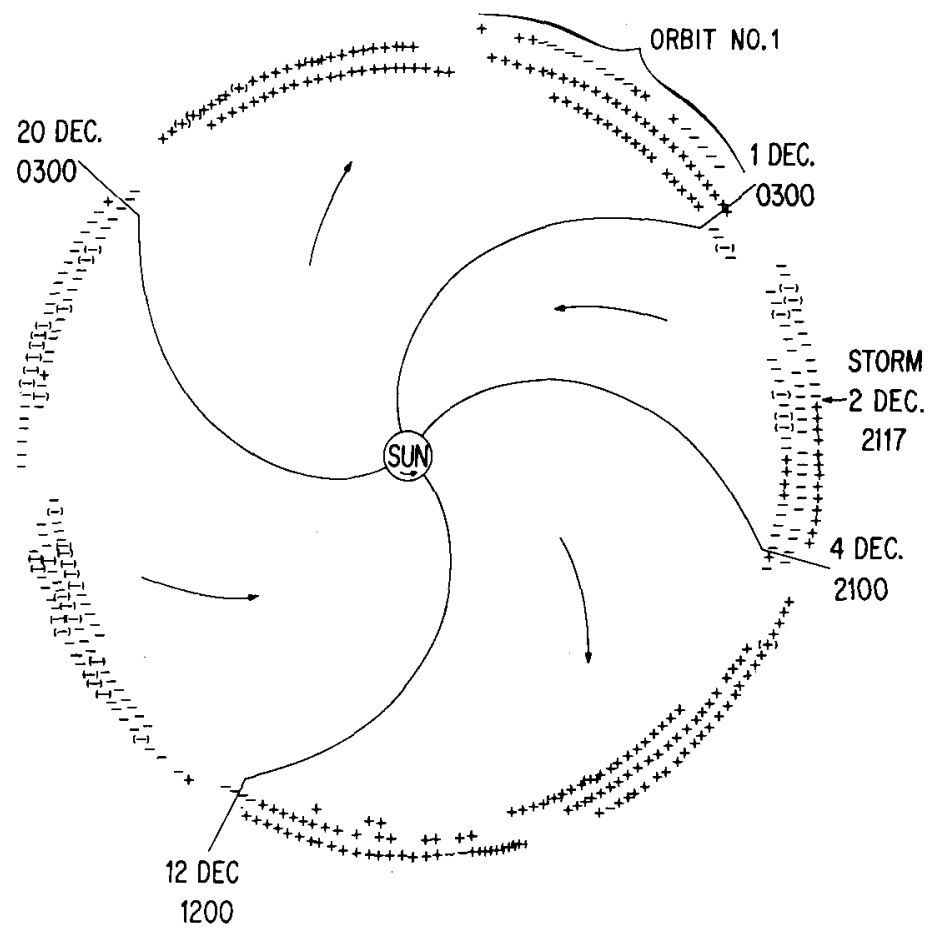

FIG. 1. Longitudinal sector structure in the interplanetary medium observed by IMP-1 in 1963-64 (Wilcox and Ness, 1965).

the solar wind velocity (see Figure 2); the field at that time was divided into two sectors per solar rotation. It should be emphasized that it is the pattern of field and plasma structures that tends to corotate with the Sun; the actual motion of the plasma is approximately in the radial direction.

The evolution of the interplanetary sector pattern during several years is shown in Figure 3. The Mariner-2 and IMP-1 patterns previously mentioned can be seen, as well 


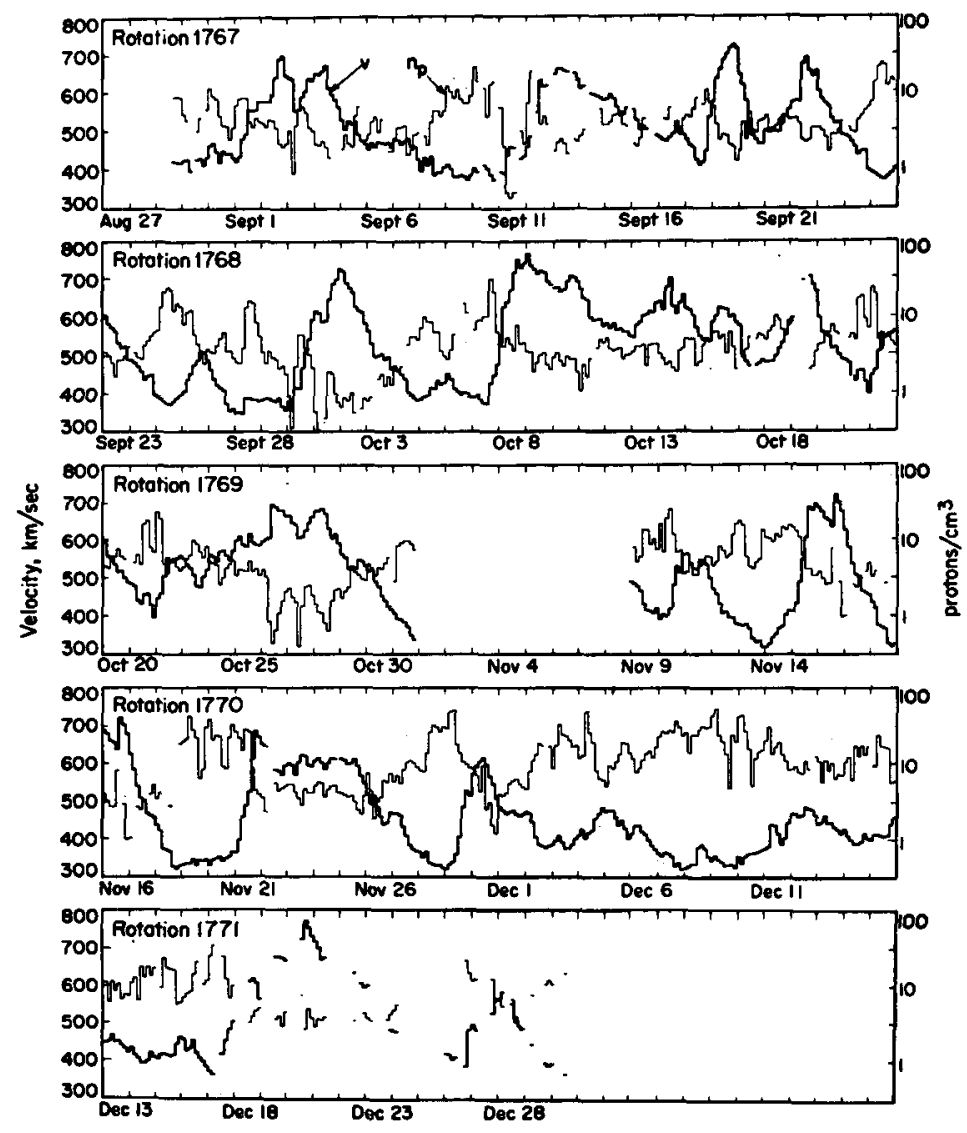

FIG. 2. Solar wind plasma velocity and proton-number density observed by Mariner-2 in 1962; plotted on a calendar of Bartels 27-day solar rotations to show recurrence properties (Neugebauer and Snyder, 1966).

as observations by several later spacecraft. Near the end of 1964 IMP-2 observed almost the same pattern as that of IMP-1, and the interpolation indicated in Figure 3 is based on this fact, plus the observed sequences of recurring geomagnetic activity. It is interesting that independent cosmic-ray observations by the Vela satellites (Asbridge et al., 1967) in the middle of 1964 also led to the conclusion that the IMP-1 sector pattern was essentially unchanged at that time.

The sector pattern shown in Figure 3 appears to be dominated by the old sunspot cycle up to the end of 1964 and the beginning of 1965, and by the new cycle thereafter. Characteristics of the old cycle pattern include a quasi-stationary structure for at least 1 year, and a recurrence interval of 27 days. Comparison of this pattern with the direction of photospheric magnetic fields has suggested that the average heliographic latitude of the interplanetary field observed near the Earth was about $15^{\circ} \mathrm{N}$ (Wilcox 


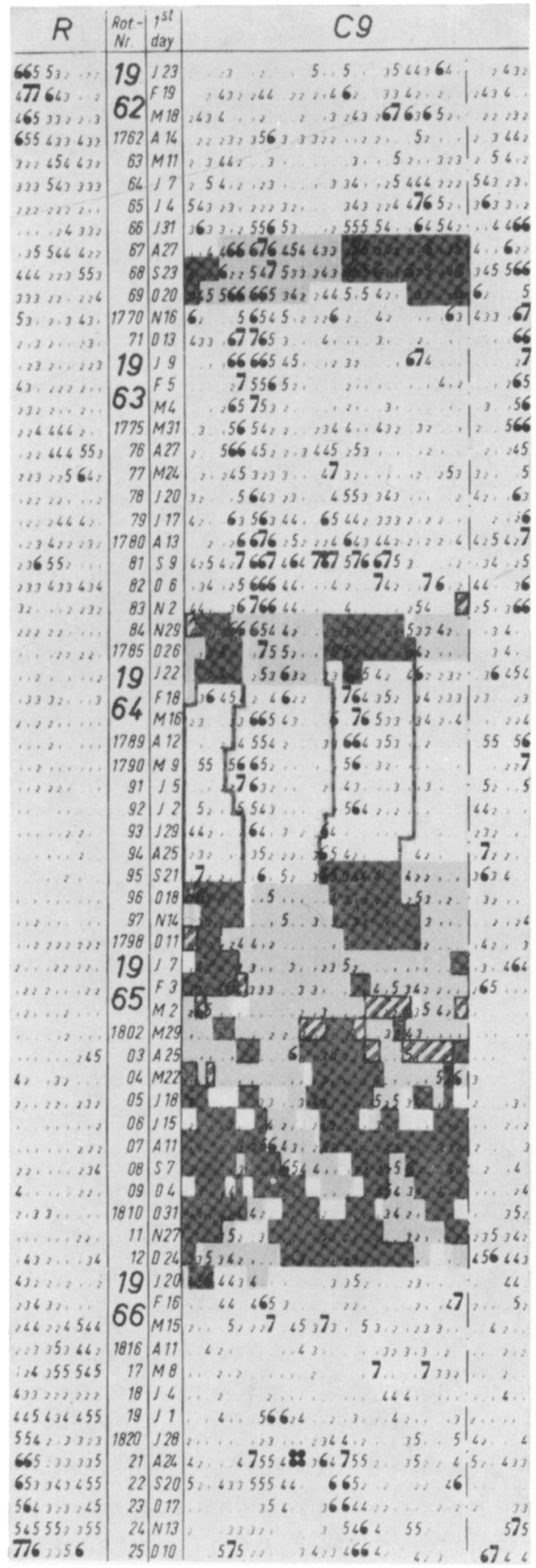

\section{MARINER 2}

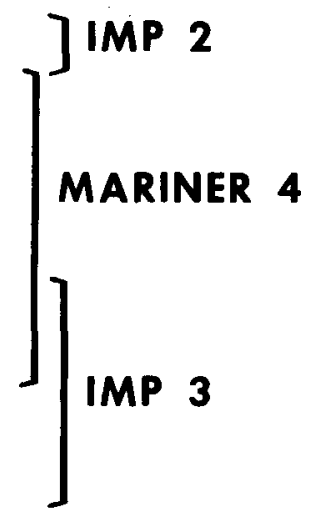

FIG. 3. Evolution of the interplanetary sector structure from 1962 to 1966, overlayed on a 27-day calendar of geomagnetic activity figure C9. Light shading is field away from Sun, dark shading is toward, and cross-hatching indicates occasional periods of mixed polarity (Ness and Wilcox, 1967). 
and Ness, 1967). This is presumably related to the overall predominance of Northern hemispheric solar activity at this time. Characteristics of the new cycle sector pattern include a more rapid evolution with time, and a recurrence interval of about 28 days. This probably indicates a source at a heliographic latitude even higher that than discussed above, which might be related to the appearance of new cycle activity at higher heliographic latitudes. Although the evolution with time is more rapid, it is interesting that so far at least during the increase of solar activity the sector property has persisted; i.e., for several consecutive days the field polarity is unidirectional.

The significance of the observed interplanetary sector structure as a possible indicator of a fundamental large-scale solar organization cannot yet be evaluated. It seems possible that although individual photospheric field elements at various latitudes respond to the differential rotation, as would be expected (and as they have been observed to do by Wilcox and Ness, 1966), an underlying pattern of the appearance of photospheric magnetic regions exists for at least several years and is related to the interplanetary pattern shown in Figure 3. A related possibility has been suggested by Dodson-Prince and Hedeman (1967) from a study of centres of activity; they also suggest that the tendency for active regions to develop on opposite sides of the Sun approximately $180^{\circ}$ away may be significant.

\section{Solar Cosmic Rays}

Several spacecraft observations of energetic particles have been interpreted in relation to the interplanetary magnetic fields. Observations shown in Figure 4 by Simpson and colleagues on IMP-1 showed that a recurring stream of protons of about $1 \mathrm{MeV}$ energy was contained within one of the sectors shown in Figure 1. The proton intensity followed the usual sector structure of a maximum in the preceding portion of the sector and a decline in the following portion. Understanding the physical processes which have populated this one sector with an appreciable intensity of recurring energetic protons is surely one of the most challenging problems for solar physics.

Our knowledge of the so-called solar cosmic rays has been considerably increased by the elegant experiments of McCracken and colleagues on Pioneers-6 and -7 in 1966. Directional properties of the cosmic radiation have been investigated utilizing a detector that rotates with the spinning spacecraft, whose axis is perpendicular to the ecliptic. The detector responds primarily to particles whose motion is approximately parallel to the ecliptic. The first particles to reach the spacecraft from a solar flare are often very much guided along the interplanetary field lines, so that the observed distribution is quite anisotropic, as shown in Figure 5, which shows the intensity of cosmic rays in the energy interval $7 \cdot 5-45 \mathrm{MeV}$ observed after three flares. If the flare position is near the middle of the Western hemisphere of the visible disk the particles tend to have a rapid Sun-Earth transit. If the flare is in the Eastern hemisphere the particles tend to arrive after a considerably longer delay. McCracken $e$ al. (1967) interpret this 

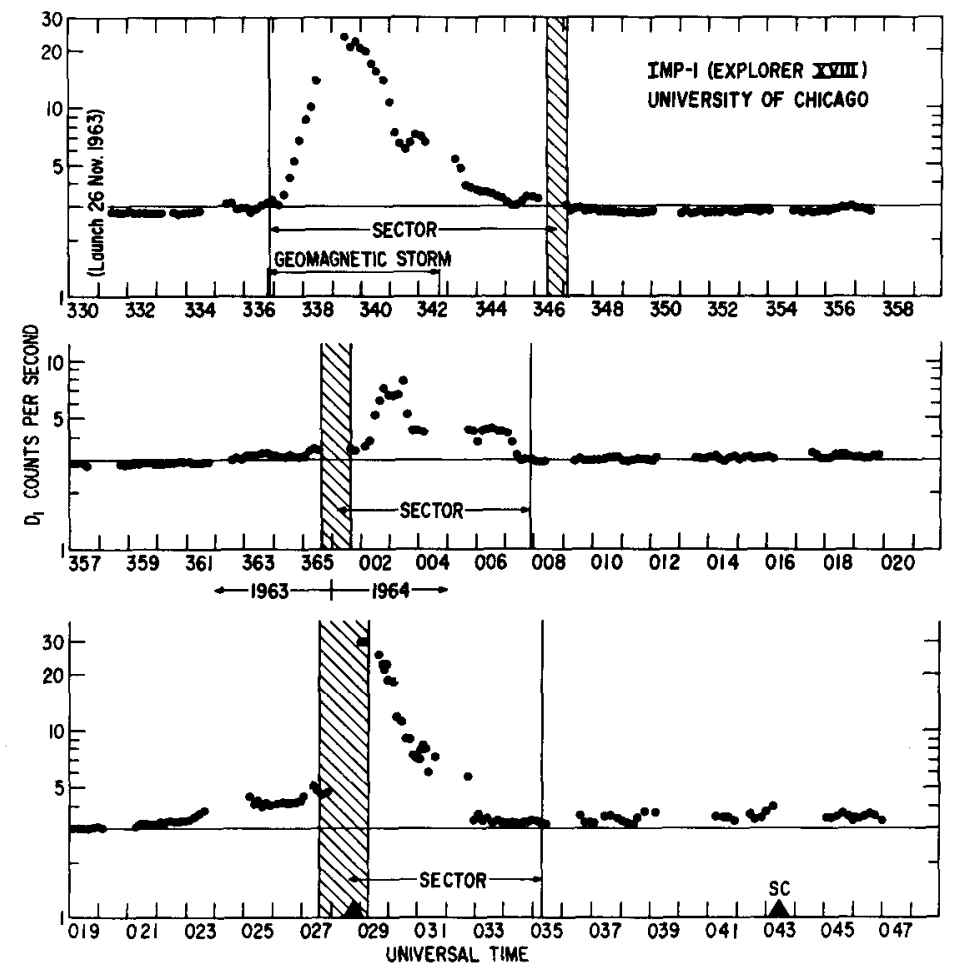

Fig. 4. Recurrent streams of 1 MeV protons observed by Simpson on IMP-1 tend to be contained within a single sector of the structure shown in Figure 1. Three 27-day solar rotations are shown (Wilcox and Ness, 1965).

as being caused by longitudinal diffusion of the flare particles close to the Sun, until they reach the magnetic lines that connect to the spacecraft and propagate rapidly along them. The mean free path for scattering in the extended interplanetary medium is estimated at $1 \mathrm{AU}$ by McCracken et al. (1967), and they suggest that earlier discussions of diffusion in this medium may need to be reexamined.

The guiding of solar cosmic rays by the interplanetary magnetic field has been demonstrated by McCracken and Ness (1966), as shown in Figure 6, which compares Pioneer-6 observations of the field direction with the direction of cosmic-ray anisotropy. The field direction observed during 4 hours is shown as the continuous line, while the superposed arrows indicate the direction of cosmic-ray anisotropy (the direction from which the maximum flux is incident on the detector). The guiding of the cosmic rays around several sharp bends in the field is obvious. It is often observed that when the field direction has an abrupt change, the cosmic-ray flux may abruptly increase or decrease. This leads to the concept of discrete filaments of magnetic flux that extend from regions very close to the Sun out to $1 \mathrm{AU}$ (and beyond). Simultaneous observations from two spacecraft at different longitudes by Ness (1966) have 

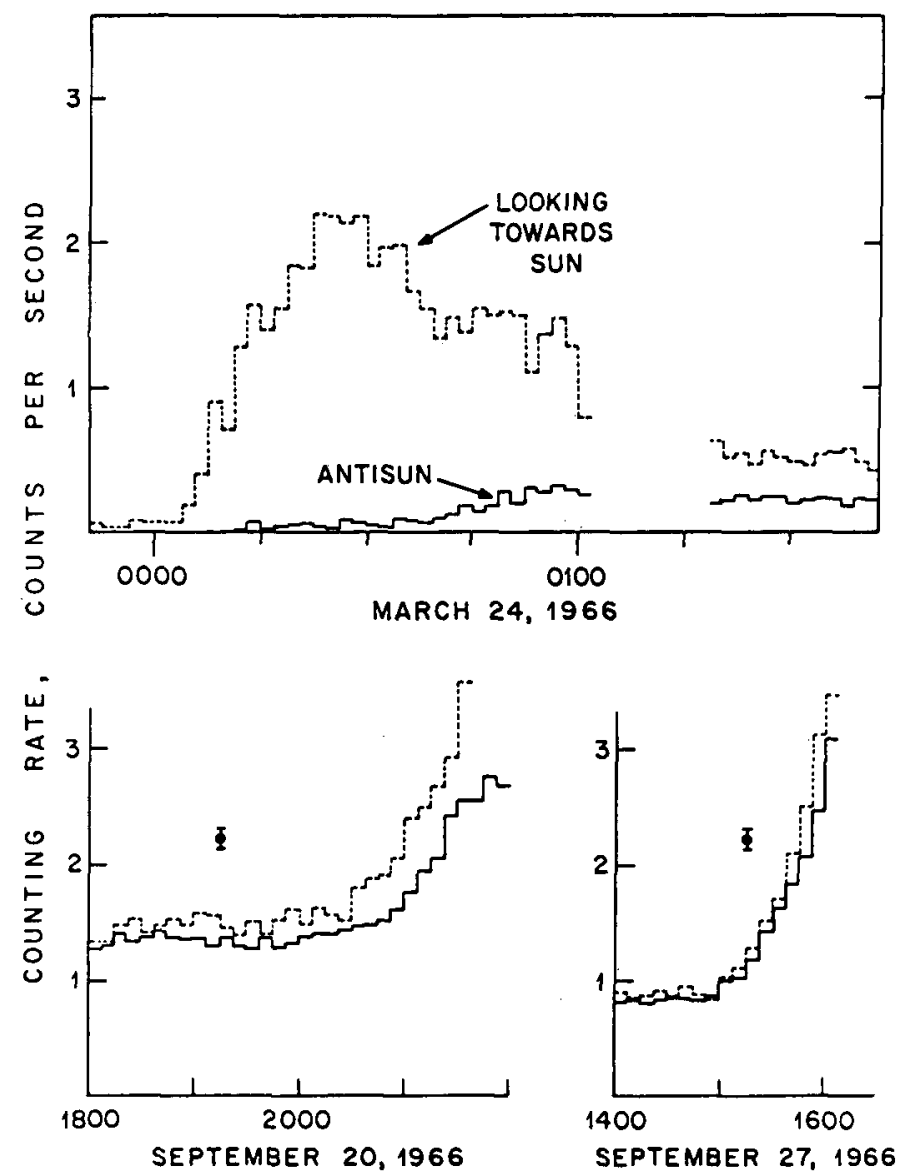

FIG. 5. Onset characteristics of three flare effects in quadrants looking toward the Sun and away for energy range 7.5-45 MeV, observed on Pioneers-6 and -7 (McCracken et al., 1967).

shown that these filaments corotate with the Sun in the same way as do the sectors previously discussed. The cross-section of these filaments is typically in the range of $0.6-5 \times 10^{6} \mathrm{~km}$. Thus at least at times there may be a detailed mapping of small-scale features close to the Sun onto a sphere of radius 1 AU. The study of the Sun through observations of interplanetary fields and plasmas by spacecraft at $1 \mathrm{AU}$ can perhaps be compared with a field-emission microscope, in which the structure of field-induced emission from a microscopic point is magnified onto a large concentric observing sphere.

\section{Solar Wind Plasma}

Recent observations in the Vela satellite program by the Los Alamos group have yielded important new information about the solar wind plasma. These satellites are 


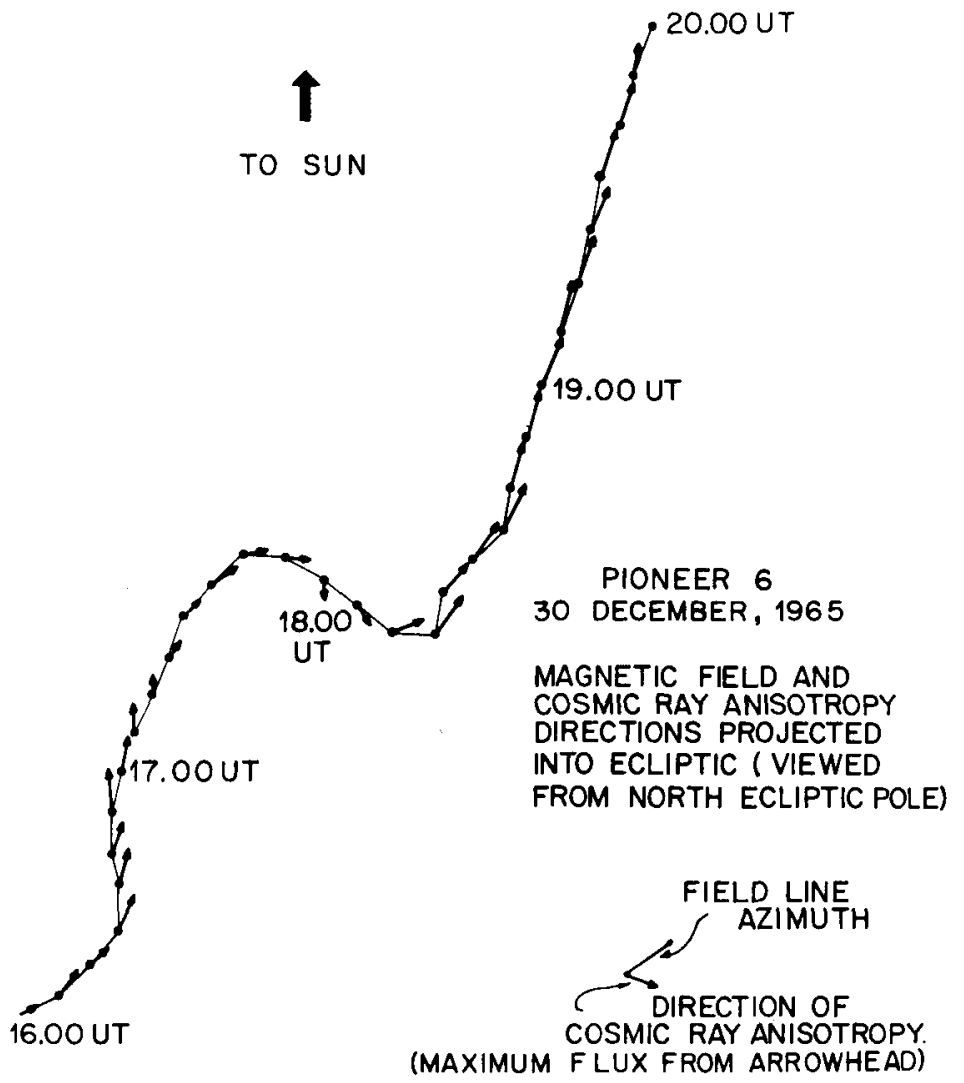

FIG. 6. Comparison of direction of interplanetary magnetic field and direction of arrival of maximum cosmic-ray flux (McCracken and Ness, 1966).

in circular orbits about the Earth at a distance of 18 Earth radii, and spend about one third of each orbit outside the magnetosphere and magnetosheath. A hemispherical plate electrostatic analyser makes detailed measurements of the incident charged particle flux. Figure 7 shows a typical spectrum and angular distribution. The large peak in the spectrum is the almost monoenergetic stream of protons with energy of slightly less than $1 \mathrm{keV}$. The small peak at the right is attributed to alpha particles having the same streaming velocity as the protons and therefore twice the energy per unit charge. The angular distribution shows that the particles are coming not quite from the Sun but rather from a few degrees East of it. Since the peaks in Figure 7 have a finite width it is clear that in addition to the streaming energy of the plasma there exists a random energy, which is often described in terms of a 'temperature', whose value has ranged from about $5 \times 10^{3} \mathrm{~K}$ to a few times $10^{5} \mathrm{~K}$, with a normal proton temperature of a few times $10^{4} \mathrm{~K}$ (Strong et al., 1966). A complete measurement of flux as a function of energy and angle is performed in about $4 \mathrm{~min}$, and a typi- 

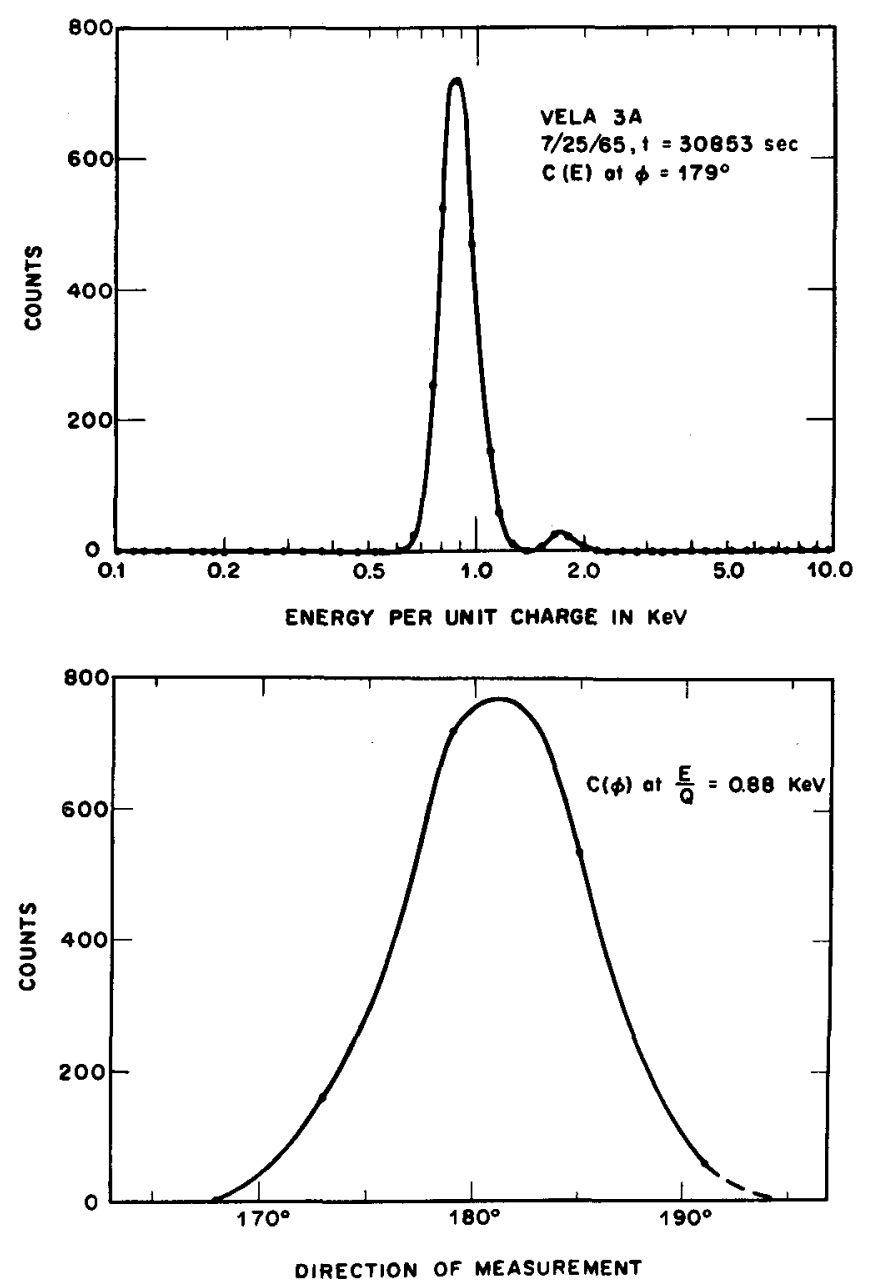

Fig. 7. Typical Vela-3 spectrum (counts vs, energy per unit charge in a fixed direction) and the associated angular distribution (counts vs. direction at a fixed energy per unit charge). The direction away from the Sun is labeled $180^{\circ}$, and the peak is a few degrees East of this (Hundhausen et al., 1967a).

cal result is presented in Figure 8. Contours of observed particle flux are shown as a function of two velocities; the abscissa is the radial velocity $V_{1}$ from the Sun and the ordinate is a velocity perpendicular to both $V_{1}$ and to the spacecraft spin axis, which is tipped by $55^{\circ}$ to the ecliptic. The arrow shows the direction of the interplanetary magnetic field simultaneously measured on the near-by IMP-3 satellite.

The contours in Figure 8 depart from isotropy in two ways. First, the temperature parallel to the field is considerably larger than the transverse temperature, the ratio being about 3 in Figure 8 , which is a typical value. Second, the temperature $(9 \cdot 2 \times$ $10^{4} \mathrm{~K}$ ) along the field in the direction away from the Sun is larger than in the field 


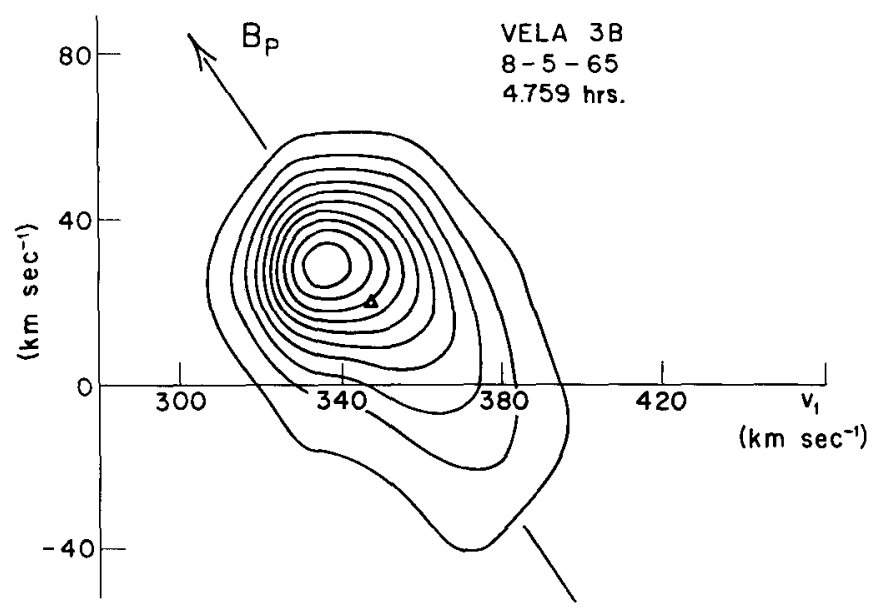

FIG. 8. Typical Vela-3 contour map of proton-velocity distribution in the radial direction from the Sun (abscissa) and in a normal direction (ordinate). The arrow is the field direction simultaneously measured on the nearby IMP-3 satellite (Hundhausen et al., 1967b).

direction toward the Sun (this is observed to be true independently of whether the polarity of the field lines is toward or away from the Sun). Thus the solar wind protons are transporting heat away from the Sun at a rate which is estimated at about $10^{-5}$ ergs $/ \mathrm{cm}^{2} / \mathrm{sec}$. A similar heat transport by electrons would be expected to be several orders of magnitude larger.

The persistence of the alignment between the plasma anisotropy direction and the field direction during a period of 6 hours is shown in Figure 9. The abrupt change in

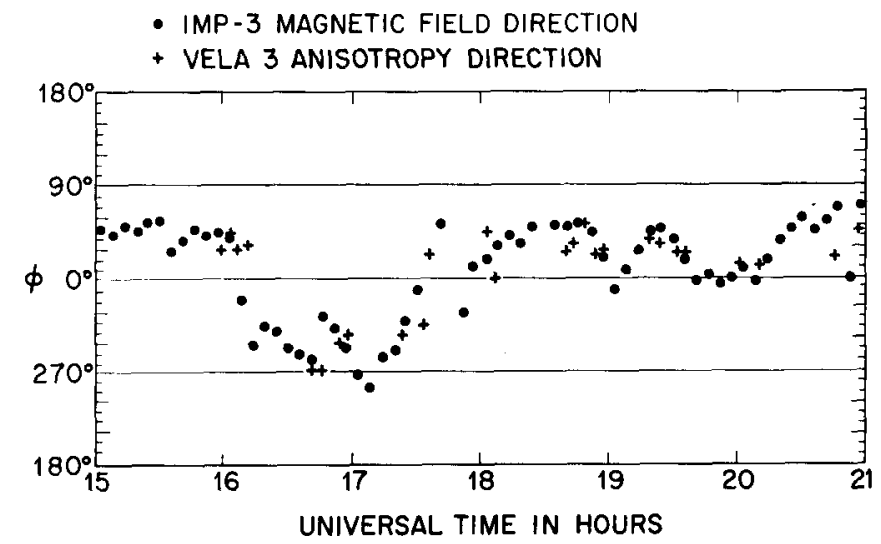

FIG. 9. The magnetic-field direction measured on IMP-3 and the direction of maximum plasmathermal anisotropy measured on Vela-3 (Hundhausen et al., 1967b). 
direction at 1600 UT probably represents the appearance of an interplanetary filament of the sort discussed previously in connection with the cosmic-ray observations of McCracken and Ness (1966). It is worth noting that during the passage of this filament past the satellite there was no significant change in any of the usual plasma parameters (mean velocity, density, temperature and alpha/proton-density ratio). Only the orientation of the anisotropic temperature distribution and the field direction changed during the passage of this filament (Hundhausen et al., 1967b).

\section{Solar Wind Azimuthal Velocity}

The solar wind angular distribution in Figure 7 shows that the solar wind was coming from a direction a few degrees East of the Sun. Individual observations show longitudinal deviations from radial plasma flow over a range of about $\pm 10^{\circ}$, but the average longitudinal plasma velocity (in the direction of corotation with the Sun) is observed to be about $10 \mathrm{~km} / \mathrm{sec}$ (Strong et al., 1967). This is consistent with the analysis by Brandt (1967a) of the orientation of ionized cometary tails. Such values for the solar wind longitudinal plasma velocity at $1 \mathrm{AU}$ suggest that corotation of the extended coronal plasma (this must be clearly distinguished from the corotation of magnetic patterns previously discussed in this paper) extends to greater distances than had previously been considered. Dicke (1964) has shown that the loss of angular momentum by the Sun to the solar wind can be computed on the basis of rigid corotation out to a radius at which the Alfvén velocity based on the radial component of the field is equal to the streaming velocity. This is a formal statement; it does not mean that rigid corotation actually exists to this distance. Dicke obtained a typical value of 35 solar radii for this distance, which is consistent with estimates by other authors (Pneuman, 1966; Weber and Davis, 1967; Modisette, 1967; Brandt, 1967b). Brandt (1966) has shown that the angular momentum loss in the solar wind could be important in the structure and evolution of the Sun.

\section{Summary}

In summary, we now have a picture of the streaming solar wind at $1 \mathrm{AU}$ carrying with it much detailed information about the solar structure. The interplanetary magnetic field is of crucial importance even though its energy density is about two orders of magnitude less than the streaming-energy density of the plasma. The interplanetary sector structure is evident in the polarity patterns of the field. Thermal anisotropies in the plasma are field aligned. Cosmic rays from localized regions of the solar atmosphere are guided to $1 \mathrm{AU}$ (and beyond) by magnetic filaments, whose pattern corotates with the Sun. The plasma velocity at $1 \mathrm{AU}$ has a small component in the direction of corotation, leading to important consequences for the extent of corotation near the Sun, the loss of angular momentum, and the structure and evolution of the Sun. 


\section{References}

Asbridge, J.R., Bame, S. J., Felthauser, H.E., Gosling, J.T. (1967) 48, 172.

Brandt, J.C. (1966) Astrophys. J., 144, 1221.

Brandt, J.C. (1967a) Astrophys. J., 147, 201.

Brandt, J.C. (1967b) Astrophys. J., 148, 905.

Dicke, R.H. (1964) Nature, 202, 432.

Dodson-Prince, H., Hedeman, E.R. (1967) IQSY/COSPAR Symposium, London, July.

Hundhausen, A.J., Asbridge, J.R., Bame, S.J., Gilbert, H.E., Strong, I.B. (1967a) J. Geophys. Res., 72, 81.

Hundhausen, A.J., Bame, S.J., Ness, N.F. (1967b) J. Geophys. Res., 72, 5265.

McCracken, K.G., Ness, N. F. (1966) J. Geophys. Res., 71, 3315.

McCracken, K. G., Rao, U. R., Bukata, R.P. (1967) J. Geophys. Res., 72, 4293.

Modisette, J.L.(1967) J. Geophys. Res., 72, 1521.

Ness, N.F. (1966) J. Geophys. Res., 71, 3319.

Ness, N.F. (1967) IQSY/COSPAR Symposium, London, July.

Ness, N.F., Wilcox, J.M. (1967) Solar Phys., 2, 351.

Neugebauer, M., Snyder, C.W. (1966) J. Geophys. Res., 71, 4469.

Parker, E.N. (1963) Interplanetary Dynamical Processes, Interscience Publishers, New York.

Pneuman, G.W. (1966) Astrophys. J., 145, 800.

Strong, I.B., Asbridge, J. R., Bame, S.J., Heckman, H.H., Hundhausen, A.J. (1966) Phys. Rev. Letters, 16, 631.

Strong, I.B., Asbridge, J. R., Bame, S.J., Hundhausen, A. J. (1967) Trans. Amer. Geophys. Union $48,182$.

Weber, E.J., Davis, L., Jr. (1967) Astrophys. J., 148, 217.

Wilcox, J.M., Ness, N.F. (1965) J. Geophys. Res., 70, 5793.

Wilcox, J.M., Ness, N.F. (1966) Astrophys. J., 143, 23.

Wilcox, J.M., Ness, N.F. (1967) Solar Phys., 1, 437.

\section{DISCUSSION}

L.W. Acton: What were the cosmic-ray energies on the last slide?

J.M. Wilcox: These particular measurements are around $15 \mathrm{MeV}$, but in general the range is a few MeV.

R. Michard: What is the relationship between energy and electric charge in the solar wind? Do you think the ratio of the two peaks agrees with the solar abundance of hydrogen and helium?

J.M. Wilcox: It would certainly seem to be related. The average ratio, over many observations is approximately $10 \%$, which is a value one would be willing to accept. However, there are considerable variations, which may be an important clue to the dynamic processes involved in the acceleration processes in the solar wind. 